\title{
Mild hypoxia is associated with quantitative EEG changes, but not with dissociative symptoms
}

F Pretorius, MB ChB, MMed (Psych), FCPsych (SA) H W Smith, MB ChB, MMed (Psych), FCPsych (SA) C Kruger, MB ChB, MMed (Psych), MD Department of Psychiatry, University of Pretoria

C Le Roux, MB ChB, MS (Aerospace Medicine) Department of Aerospace and Baromedicine, University of Pretoria

M J van der Linde, BSC, BSC Hons, PhD, UED H T Groeneveld, DSC

Department of Statistics, University of Pretoria

P Bartel, PhD

Department of Neurology, University of Pretoria

Backround and aims. Hypoxia at altitude may lead to mental changes resembling dissociative symptoms. This study examined whether hypoxia precipitates dissociative states in normal subjects and whether quantitative electroencephalographic (EEG) changes occur.

Methods. Dissociative symptoms and EEG changes were examined in a hypobaric chamber.

Results. No dissociation was noted. EEG slowing accompanied hypoxia, replicating previous findings.

Hypoxia at high altitude in aviation or mountaineering may lead to changes in mental state, including dense amnesia, psychomotor slowing, euphoria, inappropriate affect, disorientation, hallucinations or inappropriate behaviour.' The subject is often unaware of these changes and may become oblivious to danger. For example, there have been several accidents in the Drakensberg mountains involving light, unpressurised aircraft being piloted without supplemental oxygen equipment. These are labelled 'controlled flight into terrain' (CFIT) accidents and are mostly ascribed to 'bad weather' or 'human error'.

The question arose whether the abovementioned symptoms represent dissociation. Dissociative symptoms are mental symptoms where the usually integrated functions of consciousness, memory, identity and perception of the environment are disrupted. For example, a person's body or surroundings may feel unreal (depersonalisation and derealisation respectively). Dissociation may be a normal phenomenon; some individuals have a greater tendency to dissociate. Dissociation might impair decision making. We hypothesised that a dissociative tendency may predict dissociative states under hypoxic conditions.

Electro-encephalographic (EEG) changes have been described as possible neurophysiological correlates of mental symptoms and/or hypoxia. Kraaier et al. ${ }^{2}$ refer to Berger's first description in 1934 of EEG slowing during cerebral hypoxia. The first quantitative EEG analysis during hypoxia was done by Kraaier et al. ${ }^{2}$ in 1988. Hypoxia resulted in a significant increase in slow EEG activity. ${ }^{2,3}$ Concerning mental symptoms, some EEG studies demonstrated EEG slowing in patients with prominent dissociative symptoms. ${ }^{4}$

This study was designed to examine whether hypoxia may precipitate dissociative phenomena in normal subjects and whether concurrent EEG changes occur.

\section{Methods}

Twenty-three student volunteers aged 22 - 32 years participated $(10$ women and 13 men). Participants were healthy, as determined by history and appropriate physical examination, and had no ear, nose and throat (ENT), medical, neurological or psychiatric problems. They were all acclimatised to the Pretoria altitude of 4800 feet.

Subjects completed the Dissociative Experiences Scale (DES) that measures the usual frequency of dissociation. EEG recordings were made inside the hypobaric chamber at the Institute of Aviation Medicine, Pretoria. Measurements were taken under experimental conditions at 'nearly ground level' labout 6000 feet) and a simulated altitude of 12000 feet (about the altitude at the top of the Drakensberg). Each of the 2 sets of measurements 
consisted of a digital EEG recording, completion of the State Scale of Dissociation $(S S D)^{6}$ that measures the intensity of dissociative symptoms at the time, and oxygen saturation using a pulse oximeter. Subjects were kept blinded to their present altitude. Subjects spent a total of 45 minutes in the hypobaric chamber. All reasonable attempts were made to limit EEG artefacts caused by muscle movements, despite the physical constraints imposed by performing the EEGs inside a hypobaric chamber not designed for the purpose. Since drowsiness may also cause EEG slowing, subjects were monitored for any signs of drowsiness during the procedures and were aroused by an auditory stimulus if this occurred. During EEG analysis all EEG data displaying features of drowsiness were discarded.

The EEG data consisted of visual and spectral analyses (EEGFOCUS version 2.0 software, MEGIS Software, GmbH Munich, Germany). The visual EEG interpreter was blinded to the experimental conditions from which the data were obtained. Left parieto-occipital (P3-O 1) and right parieto-occipital (P4-O2) leads were used for spectral analysis. These leads had minimal artefact. Alpha rhythm is maximal posteriorly, and is relatively sensitive for the detection of slowing. Ozaki et al. ${ }^{7}$ previously described topographic EEG changes during similar conditions, which also demonstrated maximal slowing posteriorly.

\section{Analyses and results}

Mean oxygen saturation decreased significantly from $95.9 \%$ lat 6000 feet) to $88.7 \%$ (at 12000 feet) (univariate procedure, signed rank test, $p=0.0005$ ), demonstrating valid experimental conditions.

Total SSD scores did not increase at altitude, i.e. the subjects did not dissociate during mild transient hypoxia (univariate procedure of the mean difference between SSD1 (0.40) and SSD2 (0.33) scores at 6000 and 12000 feet, signed rank test, $p=0.3223$ ). Subjects did show a non-significant increase in amnesia symptoms from 0.21 to 0.29 (a subscale of the SSD) (ttest procedure, $p=$ 0.09211

The subjects' mean DES score was 9.17, which is within normal limits. 5,6 Furthermore, the subjects' dissociative traits (DES scores) were not predictive of their dissociative states (SSD scores) at altitude (regression analysis, analysis of variance (ANOVA) between the mean DES and the mean difference on the 2 SSD measurements, $p=0.4736$ )

Visual analyses of all EEGs were unchanged during mild transient hypoxia. On spectral analysis, theta activity in the occipito- parietal leads increased significantly bilaterally during hypoxia lunivariate procedure, signed rank test, $p=0.0029$ on the right, $p=0.0098$ on the left). The association between theta activity and amnesia symptoms was not significant (canonical correlations of the amnesia subscales of the SSD and theta activity on the right, $p=0.3890$, and left, $p=0.8198$ ). There was a significant correlation between theta activity on the right and hypoxia (Pearson's correlation coefficient $=-0.60627, p=$ $0.0366)$

\section{Discussion}

This study followed an integrated approach by exploring both phenomenology (dissociative symptoms) and physiology (EEG and saturation changes), reflecting the neurobiological approach of modern psychiatry.

Although the mild decrease in saturation in our study was highly significant, it did not cause dissociation. The answer to our initial question is therefore that the mental state changes found at altitude do not represent dissociative symptoms in normal subjects. Furthermore, subjects' dissociative tendency, as measured by the DES, was not predictive of dissociative symptoms during hypoxia. In our study the strongest finding was actually the significant association between theta activity on the right and mild hypoxia, replicating findings of previous studies. ${ }^{2,3}$

Nevertheless, we noted a non-significant increase in the amnesia subscale of the SSD. However, amnesia is not specific to dissociation; it is also a feature of cognitive dysfunction. The question arose whether amnesia rather indicated cognitive dysfunction in our study. Previous studies, described by Lishman,' consistently found impairment in cognitive functioning during hypoxic hypobaric conditions. It is a limitation of our study that no specific cognitive tests were administered, only a subscale of the SSD, which has not been validated as a cognitive measure. Future studies should include testing of attention, concentration, working memory, continuous performance and reaction time.

With this clear negative finding, this study does not support our hypothesis that dissociation might be a cause of the mental state changes that occur during hypoxia in normal subjects. Subjects with a history of dissociation or susceptible to dissociative phenomena were excluded from this small sample of healthy volunteers. It therefore remains to be established whether hypoxia may evoke dissociative phenomena in individuals susceptible to dissociation. We can only conclude that mild hypoxia causes slight EEG slowing in normal subjects, which is not proven to affect flight safety adversely. 
We gratefully acknowledge the staff at the Institute of Aviation Medicine in Pretoria, specifically Captain G Muller, Sergeant Z Botha and Regimental Sergeant Major $H$ Janse van Rensburg, for their assistance in operating the hypobaric chamber and the digital EEG recordings.

\section{References}

Lishman WA Organic Psychiatry. The Psychological Consequences of Cerebral Disorder. 3rd ed. Oxford: Blackwell Scientific Publications, 1997: 548-550.

2. Kraaier V, Van Huffelen AC, Wieneke GH. Quantitative EEG changes due to hypobaric hypoxia in normal subjects. Electroencephalogr Clin Neurophysiol 1988, 69: $303-312$
Schellart NAM, Reits D. Transient and maintained changes of the spontaneous occipital EEG during acute systemic hypoxia. Aviat Space Environ Med 200 1; 72: $462-470$

4. Spiegel D, Vermutten E. Physiological correlates of hypnosis and dissociation. In: Spiegel D, ed. Dissociation: Culture, Mind, and Body. Washington: American Psychiatric Press, 1994.

Bernstein EM, Putnam FW. Development, reliability, and validity of a dissociation scale. J Nerv Ment Dis 1986; 174: 727-735.

6. Krüger C, Mace CJ. Psychometric validation of the State Scale of Dissociation (SSD) Psychology and Psychotherapy: Theory, Research and Practice (formerly BrJ Med Psychol) 2002; 75(1): 33-51

Ozaki H, Watanabe S, Suzuki H. Topographic EEG changes due to hypobaric hypoxia at simulated high altitude. Electroencephalogr Clin Neurophysiol 1995 94: $349-356$

\section{$\underset{\substack{\text { EISIVIIER } \\ \text { SALNULRS }}}{\text { D. }}$ \\ KUMAR \\ CLARK}

CLIN ICAL MEDICINE SIXTH EDITION
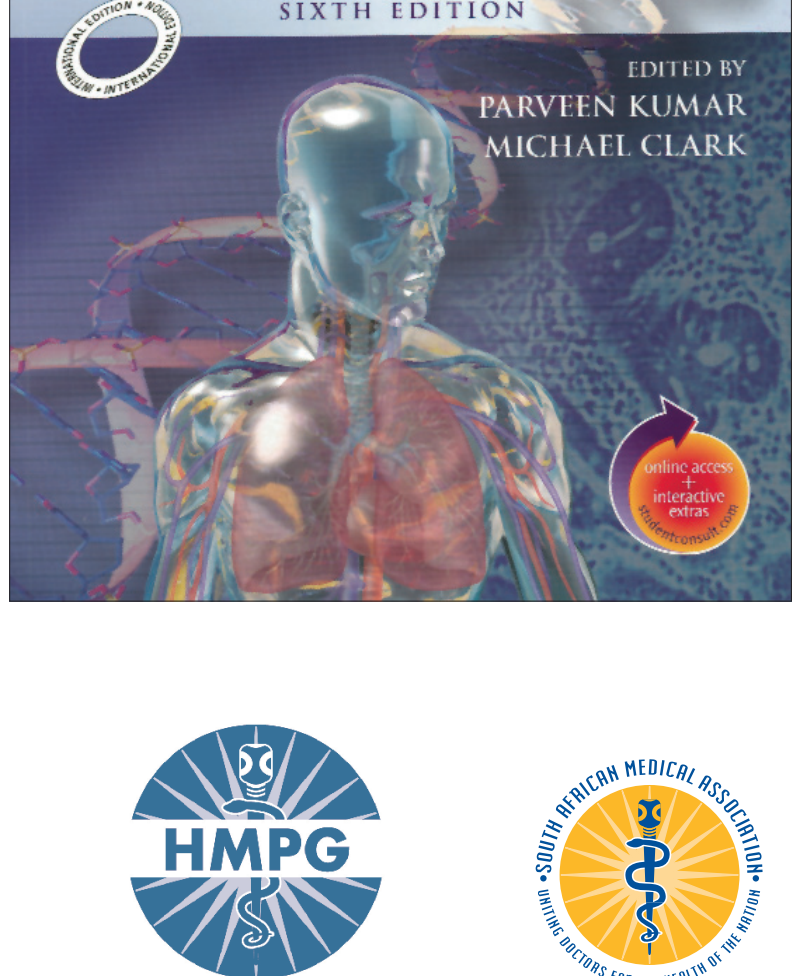

Health \& Medical Books

\section{PRICE: R380.00 SAMA MEMBERS: R360.00}

To order contact Carmen or Avril:

Tel: (021) 530-6520

Fax: (021) 531-4126 email: carmena@hmpg.co.za

or avrilm@hmpg.co.za

The South African Medical Association, Health \& Medical Publishing Group 1-2 Lonsdale Building, Gardener Way, Pinelands, 7405. 